\title{
CIRCULATING TUMOR CELLS IN BREAST CANCER: FUNCTIONAL HETEROGENEITY, PATHOGENETIC AND CLINICAL ASPECTS
}

\author{
N.V. Cherdyntseva, ${ }^{1,2,}$, N.V. Litviakov ${ }^{1,2}$, E.V. Denisov ${ }^{1,2}$, P.A. Gervas ${ }^{2}$, E.S. Cherdyntsev ${ }^{3}$ \\ ${ }^{1}$ Tomsk Cancer Research Institute, Tomsk National Research Medical Centre of Russian \\ Academy of Science, Tomsk 634050, Russia \\ ${ }^{2}$ Tomsk State University, Tomsk 634050, Russia \\ ${ }^{3}$ Tomsk Polytechnic University, Tomsk 634050, Russia
}

\begin{abstract}
Each patient has a unique history of cancer ecosystem development, resulting in intratumor heterogeneity. In order to effectively kill the tumor cells by chemotherapy, dynamic monitoring of driver molecular alterations is necessary to detect the markers for acquired drug resistance and find the new therapeutic targets. To perform the therapeutic monitoring, frequent tumor biopsy is needed, but it is not always possible due to small tumor size or its regression during the therapy or tumor inaccessibility in advanced cancer patients. Liquid biopsy appears to be a promising approach to overcome this problem, providing the testing of circulating tumor cells (CTC) and/or tumor-specific circulating nucleic acids. Their genomic characteristics make it possible to assess the clonal dynamics of tumors, comparing it with the clinical course and identification of driver mutation that confer resistance to therapy. The main attention in this review is paid to CTC. The biological behavior of the tumor is determined by specific cancerpromoting molecular and genetic alterations of tumor cells, and by the peculiarities of their interactions with the microenvironment that can result in the presence of wide spectrum of circulating tumor clones with various properties and potentialities to contribute to tumor progression and response to chemotherapy and prognostic value. Indeed, data on prognostic or predictive value of CTC are rather contradictory, because there is still no standard method of CTC identification, represented by different populations manifesting various biological behavior as well as different potency to metastasis. Circulating clasters of CTC appear to have essentially greater ability to metastasize in comparison with single CTC, as well as strong association with worse prognosis and chemoresistance in breast cancer patients. The Food and Drug Administration (USA) has approved the CTC-based prognostic test for clinical application in patients with advanced breast cancer. Prospective clinical trials have demonstrated that measuring changes in CTC numbers during treatment is useful for monitoring therapy response in breast cancer patients. Molecular and genetic analysis of CTC gives the opportunity to have timely information on emergence of resistant tumor clones and may shed light on the new targets for pathogenetic antitumor therapy.
\end{abstract}

Key Words: breast cancer, circulating tumor cells, intratumor heterogeneity, chemotherapy, liquid biopsy, prognosis of the disease course.

\section{CLONAL EVOLUTION OF TUMORS} PROVIDES INTRATUMOR HETEROGENEITY

The modern oncology paradigm was declared by Peter Nowell, who was the first to describe cancer as complex and branching evolutionary trajectories, in parallel with Darwin's iconic evolutionary speciation tree. His concept was described in 1976 in his paper entitled "Clonal evolution of tumor cell population", which was published in "Science" journal [1]. A classical or Darwinian evolutionary system embodies a basic principle: purposeless genetic variation of reproductive individuals, united by common descent, coupled with natural selection of the fittest variants [2].

Cancer is a process of a clonal evolution where sequential acquisition of mutations with concomitant, successive subclonal dominance or selective sweeps results in the tumors with various molecular

\footnotetext{
Submitted: December 06, 2016.

*Correspondence: E-mail: nvch@tnimc.ru

Abbreviations used: ALDH - aldehydedehydrogenase; BC breast cancer; CK - cytokeratin; CNV - copy number variation; CTC - circulating tumor cells; DTC - disseminated tumor cells; EGFR - epidermal growth factor receptor; EMT - epithelial-mesenchymal transition; EpCAM - epithelial cell adhesion molecule; HER2/neu - receptor to epidermal growth factor; PCR - polymerase chain reaction; TIMP1 - tissue inhibitor of matrix metalloprotease-1; TKIs - tyrosine kinase inhibitors.
}

aberrations, thus requiring personalized approach to treatment. Intratumor clonal heterogeneity, manifesting as a coexistence of tumor cells with different genotypes and phenotypes within the same tumor, is considered to be an essential driving force providing tumor clonal evolution, progression, and resistance to chemo- and radiotherapy. The clonal heterogeneity of different primary tumor sites and differences in tumor clones between primary tumors and metastases fail to provide a proper or accurate diagnosis as well as successful prognosis and treatment of cancer.

Breast cancer (BC) is the most common malignancy and is the leading cause of cancer-related mortality of women in developing countries [3]. The high BC mortality is directly endowed by the failure in early detection of the disease and the lack of effective markers to estimate the risk of cancer progression and to predict tumor response to chemotherapy and radiotherapy. Largely unsuccessful attempts to develop new approaches to prediction of response to therapy, and effective evaluation of the clinical course of BC can be mainly attributed to high intratumoral variety of this disease. Invasive carcinoma of no special type characterized by a significant intratumor morphological heterogeneity is the most common histological type of $\mathrm{BC}$, accounting for approximately $80 \%$ of all cases. We determined the tubular structures as well as trabecular, solid, alveolar, 
and discrete groups of tumor cells displaying architectural arrangement within the primary tumors $[4,5]$. The morphological structure of the primary tumor was reported to be evidently related to clinical outcome, resulting from cancer progression clinically manifested as invasion, lymph node involvement, and distant metastases consistent with results obtained from analyzing the large cohorts of BC patients, the morphological structure of the primary tumor was reported to be evidently related to clinical outcome, resulting from cancer progression clinically manifested as invasion, lymph node involvement, and distant metastases. A contribution of intratumor morphological heterogeneity to chemotherapy efficiency was also shown [4, 5]. High risk of lymphogenous metastasis was shown to be related with the presence of alveolar structures in tumors of postmenopausal women and with the increased number of different types of morphological structures in premenopausal women. The detection of alveolar structures and the greatest morphological diversity of breast tumors appeared to be associated with resistance to neoadjuvant chemotherapy [4-6].

In our study, the microarray transcriptional analysis of different morphological structures of breast tumors showed specific gene expression sets and non-coding sequences for each type of morphological structures as compared to normal breast epithelial cells. Consistent with our data, different types of morphological structures in breast tumors belong to the functionallyseparated populations of tumor cells displaying expression of various specific genes. Essential variations in the expression pattern of signaling pathways endowing tumor chemoresistance and contributing to tumor cell invasion of different structures were also shown (data are being prepared for publication).

Significant differences in the spectrum and functional activity of the major cancer-related signaling pathways in different types of the above mentioned parenchymal structures corroborate their different contribution to tumor progression and serve as the basis for the identification of new prognosis markers and targets for therapeutic intervention.

Chemotherapy may destroy certain sensitive cancer clones and erode their habitats, but it can also provide a potent selective pressure for the expansion of resistant variants. Chemotherapy and radiotherapy induce the clonal evolution of tumor cell population, leading to the expansion of dominating drug resistant tumor clones, which are considered to be the major cause of tumor progression and anticancer treatment failures. Identification of the molecular mechanisms responsible for multidrug resistance could provide the opportunity for more effective control of tumor growth and progression [7].

Tumor clone evolution is forwarded through the interaction between tumor cells and microenvironment as well as under the influence of genotoxic factors resulting in the occurrence of new mutations under conditions of high genetic instability. Tumors are composed of cells with driver changes and cell clones carrying neutral mutations, which are not selected, as well as a plurality of cells with random changes, which often may promote tumor growth. On the other hand, tumor cells can also dramatically influence to the microenvironment during evolution process, supporting tumor growth.

The primary driver mutations result in the activation of oncogenic signalling pathways and/or inactivation of tumor suppressors. They are important for tumor (carcinogenesis) and actually provide its biological behavior, determining the clinical course and outcome of the malignant process. The increased genetic instability in the primary malignant clone leads to the generation of new mutations (so-called secondary driver mutations) giving rise to new subclones, which may become dominant in the tumor over time. Passenger mutations are random single mutations that can both contribute to cancer progression and do not have any effect on the tumor behavior [8-11] (Fig. 1).
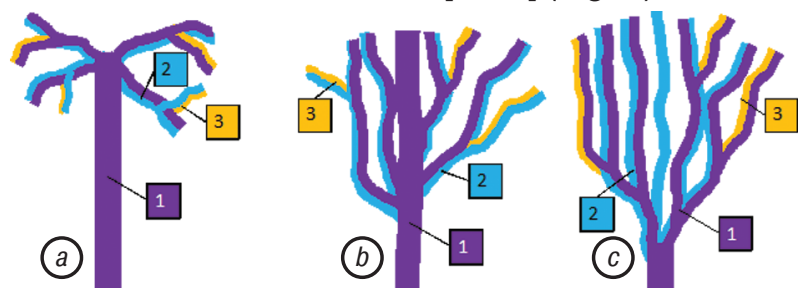

Fig. 1. Different types of tumor clonal architecture. Tumor progression is presented as a growing tree. The distribution of primary (1) and secondary (2) driver mutations as well as passenger mutations (3) within the tumor tree. Three types of tumor clonal architecture varying in the subclone amount were identified. The poor response likelihood depends on the type of tumor tree. Type "a" tumors contain more primary driver mutations and show good response to treatment. Other tumors (" $c$ ") have a high genetic diversity, manifested in a greater representation of the secondary driver mutations and mutations "passengers", and as a result, display poor therapeutic effect. Type " $b$ " tumor response to therapy has intermediate value [10-12]

The dynamics of the somatic cell evolution is dependent on the rate of mutation processes, genetic diversity and clonal expansion, and may be modified by such events as clonal interference (competition of clones having an advantage in the same adaptive environment) or parallel expansion with subsequent appearance of dominant clones [12-14]. The epigenetic alterations, which are acquired more rapidly than genetic changes, contribute significantly to the clonal evolution. They can be inherited during cell division and may determine the tumor phenotype $[15,16]$. Cancer tissue ecosystems provide the architectonic space and driving determinants for fitness selection, i.e. the so-called adaptive landscape [7, 17]. The tumor microenvironment is composed of multiple dynamically interacting components that can influence cancer clone evolution. For example, TGF- $\beta$, promoting dissemination of cancer cells through the induction of epithelialmesenchymal transition (EMT), is one of the critically important molecules regulating the tumor ecosystem, along with other inflammatory cytokines [12, 18].

The reciprocal interactions between tumor cells and the tumor microenvironment are regulated by both systemic factors (nutrients, hormones), and 
mediators, which are produced by tumor-associated fibroblasts, endothelial cells, mesenchimal progenitor cells or different types of infiltrating inflammatory cells and cancer cells itself. In each case, the external environment, lifestyle, exposures to genotoxic agents, constitutive genetics of the host cells, systemic regulators, local regulators (microenvironment) and architectural constraints provide the evolution of somatic cells, tumor biological behavior and outcome [7].

Tumor cells can re-modulate the microenvironment and create niches to endow their competitive advantage in growth and dissemination. The interaction between tumor cells and the microenvironment can be dramatically modulated by chemotherapy or radiation therapy. Although the majority of cancer cells can be killed by cytotoxic agents, the landscape remodeling creates conditions for the selection and expansion of minor variants of tumor cells insensitive to treatment [19].

Thus, each patient has a unique history of the tumor development, and to have benefit from chemotherapy, it is necessary to monitor molecular changes that can serve as both markers of acquired resistance and targets for the effects of other drugs. Chemotherapy, including targeted therapy, can be considered as the guiding factor in the cancer cell clone evolution, inducing the expansion of resistant clones instead of dead sensitive cells. For successful treatment, it is necessary to know what a new driver clone has appeared during the chemotherapy to choose a proper drug for further therapy. For example, patients with mutations in the tyrosine kinase domain of epidermal growth factor receptor (EGFR), whose tumors initially responded to chemotherapy with tyrosine kinase inhibitors (TKIs), would develop resistance to these TKIs. The acquired resistance to TKIs is provided by the appearance of additional mutations in the EGFR gene (particularly, T790M) [20] or in the chimeric gene $A L K$ [21], altering the spatial structure of the coding protein, thereby abrogating its interaction with the inhibitor.

The identification of new driver mutations promotes the development of new drugs. For example, afatinib, a recently introduced new $\mathrm{TKI}$, can be used for lung cancer patients. This inhibitor manifests its activity against T790M mutation occurring in 50\% of patients who showed resistance during the treatment with EGFR-targeted TKIs gefitinib or erlotinib [22].

The development of drug resistance phenotype, contributing to insensitivity of tumor to chemotherapy in $80-90 \%$ of cases is one of the crucial mechanisms of cancer progression [23, 24].

To estimate chemoresistance of intratumor morphological heterogeneity of $\mathrm{BC}$, we studied the expression levels of different genes, coding the ABCtransporter family, in various parenchymal structures, such as alveolar, tubular, trabecular, solid structures and individual cells. We showed the different gene expression profiles in various structures and revealed that the activation of ABC gene expression occurred most frequently in the trabecular structures [25].

In our study we also directly showed the partial destruction of tumor clones during neoadjuvant chemotherapy for BC patients, using the detection of copy number variation (CNV) as a numerical chromosome aberration, namely deletions or amplifications, in various loci. To study the CNV, microarray analysis was performed using the high density microarray platform Affymetrix (USA), CytoScanTM HD Array [26, 27]. We tested CNV in breast tumor biopsy before treatment and in surgical specimens after preoperative chemotherapy. We observed partial elimination of tumor clones carrying deletions and amplifications in a patient who had achieved clinical partial regression of the primary tumor after preoperative chemotherapy (Fig. 2). In the patient $\mathrm{E}$, whose tumor progressed while receiving chemotherapy, along with the disappearance of the clones, the emergence of new clones was observed, which appeared to provide drug resistance and tumor progression (metastasis to bones, soft tissues, lungs and cervical lymph nodes).

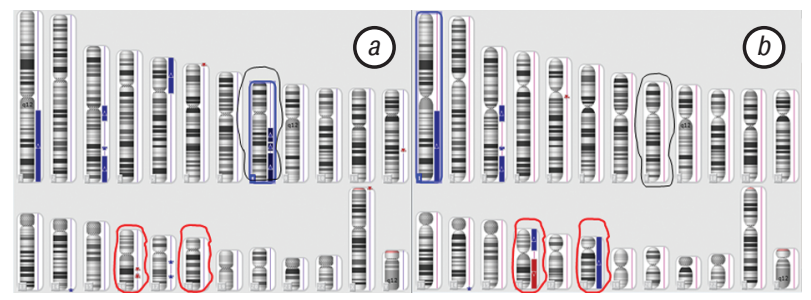

Fig. 2. Change of tumor clones during the preoperative chemotherapy in patients with BC. CNV of DNA (deletion or amplification of chromosomal regions and individual chromosomes) in breast tumor cells were determined. High density microarray (Affimetrix Cytoscan HD Array), which is able to detect the entire spectrum of mutant tumor clones was used. Red shows deletions, blue - amplification. The genetic landscape of a tumor prior (a) to treatment and after (b) treatment [27]

The biological behavior of the tumor is determined by specific cancer-promoting molecular and genetic alterations of tumor cells, and by the peculiarities of their interactions with the microenvironment. Therefore, the study of the properties of tumor cells is necessary to provide successful cancer care of patients, including diagnosis, choice of therapy, monitoring of the treatment efficacy, prognosis of disease course and prediction of therapy response.

Moreover, the molecular profiling of tumors and the development of the so-called precision oncology are becoming increasingly important in the management and therapy of cancer patients. A precision approach requires monitoring of the natural molecular evolution of individual tumors to develop the appropriate targeted therapies for each patient [28]. The personalized medicine assumes the systemic use of patient-specific genetic information (both germline and somatic) and molecular or/and cellular tumor characteristics to select the optimal treatments with the goal of improved therapeutic efficacy and reduced toxicity [29-31]. The precision medicine is considered as a general trend in the development of targeted therapy. 
CIRCULATING TUMOR CELLS: DEFINITION AND DETECTION

In clinical practice, the objective obstacles exist to obtain a sufficient amount of tumor material that can be tested at different steps of examination and treatment of cancer patients. The restrictions are caused by impossibility to perform multiple biopsies, lack of sufficient amount of tumor tissue or its complete disappearance on pathological examination in patients with complete pathological response. It is also important that the material derived from a limited number of tumor sites, may not accurately reflect the tumor heterogeneity, because the different tumor sites may contain different cell clones with specific molecular characteristics and biological behavior. This can lead to diagnostic errors and misinterpretation of molecular testing results to prescribe specific targeted therapies.

The testing of tumor properties using circulating tumor cells (CTC) and/or circulating tumor-specific nucleic acids derived from blood-sample ("liquid biopsy") is considered a promising alternative to analysis of tumor biopsy. Molecular characteristics of circulating tumor-specific DNA/RNA or CTC will enable the assessment of tumor clonal dynamics related to clinical course and detection of driver genetic changes conferring resistance to therapy. In this review we focused on CTC that gave rise to the term "liquid biopsy", proposed by the National Institute of Health (USA), as opposed to a standard tissue biopsy and later nucleic acids included in notion "liquid biopsy" [32].

However, it should be noted that the phenomenon of intratumoral heterogeneity resulted from the process of tumor clonal evolution through the interaction between tumor cells and microenvironment as well as under the influence of chemotherapeutic agents may provide the failure of "liquid biopsy" for disease prognosis and prediction of chemotherapy response.

This is due to the presence of cell clones within a tumor with different functional properties providing diversity in their biological behavior responsible for clinical course of disease (metastasis) and sensitivity (resistance) to therapy. Therefore, one can expect the coexistence of wide spectrum of circulating tumor clones with various properties and potentialities to contribute to tumor progression and response to chemotherapy. Considering the behavioral variability of distinct subpopulations of CTC in the blood we can assumed that they have different prognostic and predictive value.

In this review numerous experimental and clinical results were analyzed to show the diversity and variability in phenotype and genotype of CTC and their prognostic significance. Data on prognostic or predictive value of CTC are rather contradictory, because there are still no standard appropriate methods of CTC identification, represented by different populations manifesting various biological behavior endowing different potency to metastasis.

Tumor cells in the bloodstream are detected after the tumor removal in $30 \%$ of patients with different malignancies, including BC. They are also detected in the bone marrow of $20-60 \%$ of BC patients (socalled disseminated tumor cells - DTC). It is known that BC metastases may occur even 20 years after the treatment, and more than $30 \%$ of the patients without clinical evidence of the disease appeared to have CTC in the blood stream, indicating that tumor cells can survive in a state of dormancy for long periods [33].

Since it is known that the intravasation of tumor cells from primary tumor into the circulation is a obligatory prerequisite for metastasis [34], it is quite obvious that the presence of CTC may reflect the risk of metastatic disease in a particular patient. There are many published studies evaluating the prognostic significance of CTC as a factor of high risk for metastasis [35, 36]. However, there are also other studies that have not shown that CTCs are able to predict the risk of cancer dissemination $[37,38]$.

There are the following reasons for these controversial results: lack of a standard method of identification of CTC, lack of fundamental knowledge of the phenotypic and genetic characteristics of different CTC populations, mechanisms of metastasis, and the absence of validated information about their relation with the clinical course of the disease.

Along with single CTC, the circulating clusters or micrometastases (2-50 united cell) have been found in the blood of patients with various malignancies [39]. CTC clasters, which are responsible for the development of future metastases, are a result of the collective invasion and subsequent intravasation of tumor cells into lymphatic or blood vessels. It is assumed that their penetration into the blood vessels occurs in the areas of destruction of the endothelium [40], and is promoted by tumor cells undergoing EMT [41] as well as by cooperation with tumor-associated fibroblasts [42], eventually contributing to proteolysis of the vascular walls. Vascular endothelial growth factor has been shown to contribute to the collective cell intravasation into blood stream and accumulation of tumor clusters [43].

A recent study using a mouse model of $B C$ has shown that CTC clusters have oligoclonal origin from primary tumor cell groups, thus confirming the fact of intravasation and denying the opportunity of a random aggregation of clusters from single CTC [44]. Along with the mechanism of cluster origin by invasion of cell groups to the blood vessels, there are published data on circulating clusters organized from single proliferated cancer stem cell adhered to the endothelium [45], or by the aggregation of single cancer stem cell with blood cells, in particular with lymphocytes and platelets. A recent study confirmed that CTC are composed of tumor cells, although some cases $(<5 \%)$ demonstrated the presence of immune system cells expressing CD45, CD68, CD14 and other markers in their structure. All CTC showed a low expression of transcripts encoding epithelial CTC markers, such as keratin, mucin 1 (MUC1), epithelial cell adhesion molecule (EpCAM) and/or CDH1 [46, 47]. 
Numerous CTC detection techniques have been developed so far. The cytological CTC identification method was first proposed by Nabar in 1959. However, this method was found to be low in specificity. The most commonly used CTC detection approach is based on the use of monoclonal antibodies against epithelial markers, such as EpCAM and cytokeratin (CK), provided that CTC do not express hematopoietic cell markers. The Cellsearch System ${ }^{\mathrm{TM}}{ }^{\circledast}$ (Veridex, Warren, NJ, USA), the first Food and Drug Administrationapproved commercial automated system developed for the identification of EpCAM+, CKs 8, 18, 19+, CD45and nuclear cells, is considered as an effective tool for determining prognosis in patients with metastatic BC, prostate cancer and colorectal cancer [48].

Considering the fact that the CTC detection using different markers can identify cell subpopulations with different biological behavior, it is obvious that the Cellsearch system has significant limitations because it detects the epithelial markers only. However, it is known that EpCAM expression is downregulated during EMT, and gain of mesenchymal markers such as vimentin and fibronectin was found to correlate with a worse prognosis more effectively than CK-positive cells [49]. Recent studies show that CTC markers may change over the course of therapy [50].

AdnaTestBreast ${ }^{\mathrm{TM}}$ test is based on the CTC detection by assessing the EpCAM gene expression [37]. However, a prospective German multicenter trial (DETECT) showed no correlation between CTC and disease-free or overall survival [37].

The microfluidic platform (the "CTC-chip") deals with small blood volumes and uses antibodies against the common epithelial cell surface marker EpCAM [51]. Recently, a refined methodology called "herringbonechip", or "HB-Chip", has been developed to provide an enhanced platform for CTC isolation. The CTC chip was created for isolation of rare CTC in patients with breast, lung and prostate cancers [52].

The immunospot assay (Elispot) method is also used for detection of CTC or bone marrow DTC. Using this method, only viable tumor cells are detected and appropriate cytokine secretion is studied at the individual cell level. The reverse transcription quantitative polymerase chain reaction (PCR), is used for detecting of CTC marker gene expression, such as CK-19, mucin 1, mammoglobin, EGFR and CK-20. In the metastatic BC setting, plasma levels of the miR-200 family, as well as a few other circulating microRNAs, are highly correlated with CTC in the blood and show great potential in predicting the survival of these patients [53].

CTC isolated from blood by different methods can be assessed by immunohistochemistry for specific markers. Fluorescence in situ hybridization is used to detect gene amplification or translocations. DNA or RNA extracted from the CTC can be used for the reverse transcription quantitative PCR to assay gene expression profiling. However, the isolation of CTC with high purity as well as the getting alive cells to be cultivated in vitro is a technical problem. All these approaches focus on the detection of already known molecular changes, whereas the full genome sequencing can be used for the detection of new aberrations, and the expression profiling for the detection of previously unknown activated signaling pathways providing CTC function.

Thus, it is obvious that the efficiency of using CTC as markers depends on the detection method, as different types of CTC with different metastatic potential can be identified. Different technologies may detect different CTC subpopulation with different sensitivity and purity.

\section{PROPERTIES AND PHENOTYPE OF CIRCULATING TUMOR CELLS}

The common definition of CTC designate them as epithelial cells with characteristics of the primary tumor cells, which are able to initiate the metastasis, and therefore, they can be regarded as a marker for prediction of distant metastases [53]. However, it is only partially true, because in order to leave the primary tumor, cancer cells must acquire certain properties, namely: locomotor phenotype due to EMT, ability to survive in the bloodstream, avoiding anoikis (cell death induced by the loss of attachment to the epithelial cell layer), resistance to chemotherapy and immune-mediated death in the bloodstream. For this, the clonogenic potential and ability to return to the epithelial phenotype (mesenchymal-epithelial transition) are important conditions for providing tumor cell homing to secondary organs and formation of macrometastasis [53].

CTC are extremely rare, estimated as one CTC per billion normal blood cells in the circulation of patients with advanced cancer. They can passively enter the blood circulation during vessel damage or surgery, as well as via an active migration process, moving away from the primary tumor and entering the blood circulation due to their conversion to the EMT phenotype. The level of epithelial E-cadherin expression decreases, while the level of neural $\mathrm{N}$-cadherin expression increases, thus resulting in the violation of cell adhesion. At the same time, the extracellular matrix is destroyed by matrix metalloproteinases and components of the urokinase plasminogen activator system. Increased expression of EMT markers has been found in the CTC and bone marrow disseminated cells. Depending on the origin of the primary tumor, CTC have different properties and different surface markers: epithelial cell markers, such as EpCAM, $\mathrm{CK}^{+}$, EMT-related genes such as vimentin, fibronectin, etc., and stem cell markers, such as $\mathrm{CD} 44^{+} \mathrm{CD} 24^{-} \mathrm{ALDH}^{+}$ (aldehydedehydrogenase). CTC can also express different molecules of major cell signaling pathways, such as EGFR, PI3K, Akt, etc. [54]. It is important to note that CTC can also colonize their primary tumors, accelerating cancer progression [55].

The activation of WNT signaling pathway involved in the regulation of cell adhesion was found in 30\% of CTC in contrast to only $1 \%$ of WNT activated cells 
found in the primary tumor [44]. The WNT pathway is known to provide inhibition of tumor cell death via anoikis, that results in their survival in bloodstream.

The co-expression of EMT markers TWIST and stem cell markers ALDH1 on circulating pan-cytokeratin positive cells was found in $30 \%$ of early BC patients and in $80 \%$ of metastatic BC patients. In a recent study, Schindlbeck et al. compared CTC enumeration with DTC detection using CellSearch technology in patients with primary or metastatic BC [56]. The authors found a significant concordance (69.4\%) between DTC and CTC, which increased in patients with metastases. Other authors identified EMT markers, such as PI3Ka, Akt-2, and TWIST1, and stem cell markers, such as ALDH1, Bmi1 and CD44 in CTCs of patients with early BC [57].

As for circulating clusters, they were characterized by the increased levels of tissue inhibitor of matrix metalloprotease-1 (TIMP1) and platelet transcripts, and were in a hybrid state of EMT when the expression of both epithelial and mesenchymal transition took place $[58,59]$. This state of EMT was reported to be characteristic of collective invasion and was associated with more aggressive cancer $[59,60]$.

Another study identified the adhesion molecule plakoglobin, as being higher expression in circulating cell cluster than in single CTC [44]. Cheung et al. reported that CTC clusters and lung metastases frequently expressed epithelial cytoskeletal protein, keratin 14 (K14). The RNA sequencing analysis revealed that $\mathrm{K} 14$ positive cells were enriched for desmosome and hemidesmosome adhesion complex gene, and were depleted for MHC class II genes. Suppression of K14 expression resulted in the inhibition of distant metastases, likely, through a violation of the activity of numerous molecular players, including tenascin C (TnC), Jagged 1 protein (Jag 1) and epiregulin (Ereg) [39].

\section{CIRCULATING TUMOR CELLS: RELATION TO METASTASIS AND THERAPY RESPONSE}

Evaluation of the prognostic significance of CTC in patients with early BC is intensively carried out. There are many reports indicating that the presence of CTC is associated with lower overall and progression-free survival rates $[61,62]$. The authors argue that EMT-associated markers in CTC predict unfavorable prognosis more effectively than epithelial markers [49]. It indicates more aggressive metastatic potential of cells carrying EMT markers due to the high potential for extravasation and subsequent adaptation in the microenvironment at secondary sites, namely premetastatic niches.

The properties of tumor cells, providing their metastatic potential, are significantly modified under the influence of microenvironment, including endothelial cells, fibroblasts, inflammatory cells (macrophages, neutrophils), and mesenchymal stem cells [63]. Tumor-associated macrophages enhance the invasive- ness of the primary tumor cells, producing epidermal growth factors. Blood platelets aggregate with tumor cells, protecting them from damage in the bloodstream. This likely explains the association between thrombocytosis and lower survival rates, and the effective use of anticoagulants to reduce the incidence of metastasis [63].

Cristofanilli et al. [64] in their study with a large cohort of $\mathrm{BC}$ patients concluded that the number of CTC was an independent predictor of progression-free survival as well as overall survival. The level of CTC of less than 5 tumor cells per $7.5 \mathrm{ml}$ of blood was associated with high rates of disease-free and overall survival, but not with tumor response to chemotherapy [64].

As it was mentioned above, a validated test for CTC detection has been approved for clinical use in patients with metastatic BC. Numerous studies report that the detection rate of CTC is significantly higher in patients with disseminated $\mathrm{BC}$ than in patients with early $\mathrm{BC}$ (50-80\% vs 5-13\%) [56]. Thus, it can be concluded that the count of CTC with metastatic potential is higher in patients with advanced stages than in patients with early stages. The sensitivity of CTC detection techniques can be reduced due to the low frequency of CTC in patients with early BC [65].

Krishnamurthy et al. [36] found no relationship between the frequency of CTC detection in patients with locally advancer $\mathrm{BC}$ and the standard prognostic factors, including hormone receptors and HER2/neu status. Circular tumor cells are believed can independently predict dissemination to different secondary sites. The detection of CTC and DTC is considered as useful in selecting patients for adjuvant chemotherapy [36].

Rack et al. [66] evaluated $1767 \mathrm{BC}$ patients for the presence of CTC and found the correlation between the CTC level and positive lymph nodes. Lang et al. [67] showed the correlation between CTC detection and bone marrow micrometastases and HER2 status of the primary tumor.

The tumors belonging to distinct molecular subtypes are known to have different responses to treatment and different clinical courses. Ignatiadis et al. [68] showed a prognostic significance of CK19 mRNA-positive CTC in patients with estrogen receptor-negative, triple-negative and HER2-positive BC. The authors reported elimination of CK 19 mRNA $^{+}$ CTC during the treatment with trastuzumab, a humanized anti-HER2 monoclonal antibody. The identification of CTC after adjuvant chemotherapy could serve as an independent predictor of tumor progression [68]. It was shown that in patients with metastatic $\mathrm{BC}$, the treatment with lapatinib significantly reduced the number of HER2-positive CTC regardless of the HER2 status of the primary tumor. This finding gives the opportunity to monitor molecular changes during the target therapy to control its efficacy [69].

The c-erbB2 (HER2) gene amplification was shown to play a critical role in the pathogenesis of human BC. The activation of c-erbB2 gene was observed 
in $20-30 \%$ of early BC. It was also demonstrated that HER2-positive CTC had a high metastatic potential [33]. The heterogeneity of HER2 status was revealed, some tumor cells had HER2/neu gene amplification and others show normal copy numbers. It is interesting to note that HER2-positive CTC were found in $89 \%$ of HER2 negative patients, and these patients had a survival benefit after trastuzumab-based therapy [70]. The CTC test may provide the additional information for determining HER2 status of the tumor and administering trastuzumab [71].

The CTC monitoring during trastuzumab treatment allows prediction of resistance to this drug and gives the opportunity of using appropriate inhibitors affecting STAT3 or PI3K/mTOR signaling pathways contributing to cancer progression. A series of studies were conducted to evaluate the response to therapy in BC patients with heterogeneity of HER2/neu expression in cells of the primary tumor and in CTC [33, 70-73].

By studying the relationship between CTC with HER2 amplification and efficiency of targeted therapy, it can be assumed that it is crucial to determine the grade of heterogeneity of the targets for specific therapy, which influence the treatment outcome. For this, large-scale clinical trials are required.

Prospective studies provided data on the efficacy of CTC detection for monitoring chemotherapy [61, 62]. Molecular studies of CTC might discover new molecular targets for treatment and predictors of poor response due to the emergence of resistant clones giving the opportunity to choose the drug. Molecular characterization of CTC may contribute to the development of novel anticancer drugs.

In the SUCCESS (Simultaneous Study of Gemcitabine-Docetaxel Combination Adjuvant Treatment, as well as Extended Bisphosphonate and Surveillance) trial, including 2026 patients with stage I-III BC, the CTC count detected before treatment using the CellSearch technology was an independent predictor of disease-free, overall and BC-specific survivals. CTC were tested during chemotherapy in 1492 patients and were detected in $22 \%$ of these patients. The median follow-up was 36 months. Recurrence was found in $28 \%$ of patients who had at least 5 CTC in the blood before the start of systemic treatment and in $7 \%$ of patients who had no CTC. A total of $14 \%$ of the CTC-positive patients died of BC compared with $3 \%$ of the CTC-negative patients. The presence of CTC both before the start of systemic adjuvant treatment and after completion of chemotherapy was associated with deteriorated survival [66].

It is known that CTC-clusters have a stronger ability to induce metastasis than the equivalent number of single CTC. This fact was reported in the early 70 -ies of the last century, when CTC-clusters and single CTC were injected to the experimental animals and the number of metastatic foci were compared [74]. A recent study confirmed that CTC clusters indicated a metastatic potential 23 to 50 times greater than single CTC, and metastases developing from clusters led to dramatically reduced survival [48].

In mouse models of $\mathrm{BC}$, experimentally aggregating tumor cells into clusters displayed a $>15$-fold increase in colony formation ex vivo and a $>100$-fold increase in metastasis formation in vivo [39]. In this study, the authors observed CTC-clusters at different stages of metastasis, including collective invasion, local dissemination, intravasation, circulation and formation of micrometastases, and they demonstrated that the polyclonal dissemination of CTC-clusters is a frequent mechanism in a common mouse model of BC, accounting for more than $90 \%$ of all metastases [39]. The recent studies demonstrate that the monitoring of CTC in combination with CTC-clusters provides a higher prognostic value in assessing the risk of metastatic spread of advanced BC compared to CTC detection alone [75].

There are also reports that CTC-clusters show higher resistance to chemotherapy than single CTC $[47,76]$. The increased metastatic potential of CTC-clusters and their resistance to chemotherapy are likely to relate with the lack of proliferating cells and the ability of cells to avoid anoikis [76, 77]. In addition, an enhanced survival advantage of CTC clusters also might be afforded by continued production of autocrine pro-migratory factors, matrix proteases and protection of cells from immunological surveillance by lymphocytes and natural-killer cells.

Several studies have shown a significant portion of tumor cells involving in EMT within the cluster cell population, thus also explaining their resistance to chemotherapy $[46,77]$. It is related to the increased activity of $A B C$ transporters in EMT-cells, which are responsible for drug resistance. There are also reports that CTC clusters contain cells with a cancer stem cell phenotype, characterized by high metastatic potential [78].

The potential of CTC to predict relapse and overall survival in early BC patients may depend on timing of blood sampling, duration of follow-up and more importantly on the method of CTC detection. The different results obtained by different authors may be due to a number of objective reasons: the nature and properties of CTC in each individual patient are not known; CTC may shift from an active state to a dormant state, being resistant to chemotherapy and immune attack; there is no clear evidence whether chemotherapy results in the increase or decrease of CTC number.

It is particularly important that the conditions for the development of macrometastases are objectively varied in different individuals. According to fundamental knowledge about the biology of tumor dissemination, the metastasis development is determined not only by the tumor cell behavior, but also the environment influencing their mobility, migration from the primary tumor and survival in the bloodstream and distant sites. Indeed, the Paget's "seed and soil" hypothesis (1889) was proposed to explain that metastasis is a result of cross-talk between selected cancer cells (the 
"seeds") and specific organ microenvironments (the "soil") [79]. This idea was later developed by Lyden into the concept of "metastatic niches" [80]. Primary tumor cells provide tumor invasion and regulate creation of pre-metastatic niches by secreting various cytokines and growth factors, which promote the release of cells from the tumor and mobilization of bone marrow cells into metastatic niches [81].

It is known that in patients with certain types of tumors, for example, with luminal subtype of BC, metastases can occur many years after initial diagnosis [38]. This is due to the phenomenon of "dormant tumor", when tumor cells extravasated in the secondary sites (pre-metastatic niches) do not proliferate and do not form macrometastases.

Initiation of secondary tumor growth is induced by the specific conditions related to various types of injury (trauma, surgery, radiotherapy, chemotherapy), other stress, and inflammatory processes that are associated with the activation of regeneration processes [82, 83].

The presence of tumor cells in the circulation and metastatic sites is assumed not to be a sufficient condition for macrometastasis, because even if CTC are present, the conditions of "soil" may not be fully taken into account. However, it should be noted that the underestimation of the effect of "soil" does not detract from the value of CTC detection, as "side effect" in this case will always be a benefit for the patient's survival, if there are no objective conditions for the development of macrometastases.

\section{CONCLUSION}

The numerous reports may justify the prospect of using the CTC detection not only in metastatic cancer, but also in the early stages of BC, thus improving efficiency of the treatment for early $\mathrm{BC}$. The fundamental knowledge about the nature of CTC, their properties, and the correlation between CTC and clinical course of disease indicate their high potential value as markers of tumor progression and targets for therapeutic intervention. High phenotypic and functional heterogeneity of CTC open the opportunity for determining the molecular profile of different CTC subpopulations to identify metastasis-related prognostic phenotype and genotype, providing the evaluation of metastatic potential.

Detection of molecular changes in CTCs during chemotherapy, resulted in chemoresistance, is the promising way to shift the course of chemotherapy right time as well as to find new therapeutic targets. The identification of CTC activated signaling pathways, contributing to clinical outcome, can provide an effective search for new drug targets. For example, the activation of WNT signaling pathway observed in 30\% of CTC vs $1 \%$ of primary tumor cells provides inhibition of anoikis, showing the potential to identify the targets for pathogenetic therapy.

CTC are increasingly recognized as the main source for recurrence and metastasis. The develop- ment of novel therapeutic techniques that target СТC includes:

- dialysis after surgery to remove CTCs from the bloodstrem [84];

- drugs that target cancer stem cells;

- targeted drugs for various subclones of CTC;

- identification and inactivation of signaling pathways that allow CTC to survive in the bloodstream and home to secondary sites.

Thus, we can conclude that further large-scale evidence-based clinical trials are needed to determine the prognostic and predictive value of CTC in BC, taking into consideration the "price/benefit" ratio.

\section{ACKNOWLEDGMENTS}

This work was supported by the Russian Science Foundation, Grant 14-15-00318, and Tomsk State University Competitiveness Improvement Program.

\section{CONFLICT OF INTEREST}

None declared.

\section{REFERENCES}

1. Nowell PC. The clonal evolution of tumor cell populations. Science 1976; 194: 23-8.

2. Cahill DP, Kinzler KW, Vogelstein B, Lengauer C. Genetic instability and darwinian selection in tumours. Trends Genet 1999; 15: M57-60.

3. Chissov VI, Starinsky VV, Petrova GV. Malignant tumors in Russia in 2011 year (morbidity and mortality). M: P.A. Gertsen Moscow Research Oncology Institute, Russian Health Ministry, 2013. 289 p.

4. Perelmuter MV, Zavjalova VM, Slonimskaya EM, et al. Conjugation of lymphogenous metastatic spread and histologic pattern of infiltrative component of ductal breast cancer. Sib Oncol J 2006; (1): 32-5 (in Russian).

5. Zavyalova MV, Perelmuter VM, Vtorushin SV, et al. The presence of alveolar structures in invasive ductal NOS breast carcinoma is associated with lymph node metastasis. Diagn Cytopathol 2013; 41: 279-82.

6. Denisov EV, Litviakov NV, Zavyalova MV, et al. Intratumoral morphological heterogeneity of breast cancer: neoadjuvant chemotherapy efficiency and multidrug resistance gene expression. Sci Rep 2014; (4): 4709.

7. Greaves M, Maley CC. Clonal evolution in cancer. Nature 2012; 481: 306-13.

8. Barcellos-Hoff MH, Park C, Wright EG. Radiation and the microenvironment - tumorigenesis and therapy. Nat Rev Cancer 2005; 5: 867-75.

9. Bardelli A, Cahill DP, Lederer G, et al. Carcinogenspecific induction of genetic instability. Proceed NAS 2001; 98: $5770-5$.

10. Gerashchenko TS, Denisov EV, Litviakov NV, et al. Intratumor heterogeneity: nature and biological significance. Biochemistry (Mosc) 2013; 78: 1531-49.

11. Cherdyntseva NV, Lytviakov NV, Denisov EV. Fundamental oncology progress in 2012. Pract Oncol 2013; 14: 1-12.

12. Hanahan D, Weinberg RA. Hallmarks of cancer: the next generation. Cell 2011; 144: 646-74.

13. Loeb LA. Human cancers express mutator phenotypes: origin, consequences and targeting. Nat Rev Cancer 2011; 11: 450-7.

14. Weisenberger DJ, Siegmund KD, Campan M, et al. $\mathrm{CpG}$ island methylator phenotype underlies sporadic mi- 
crosatellite instability and is tightly associated with BRAF mutation in colorectal cancer. Nat Genet 2006; 38: 787-93.

15. Ponomaryova AA, Morozkin ES, Rykova EY, et al. Dynamic changes in circulating miRNA levels in response to antitumor therapy of lung cancer. Exp Lung Res 2016; 42: $95-102$.

16. Ponomaryova AA, Cherdyntseva NV, Dobrodeev AY, et al. RAR $\beta 2$ gene methylation level in the circulating DNA from blood of patients with lung cancer. Eur J Cancer Prevent 2011; 20: 453-5.

17. Gatenby RA, Gillies RJ. A microenvironmental model of carcinogenesis. Nat Rev Cancer 2008; 8: 56-61.

18. Bierie B, Moses HL. Tumour microenvironment: TGF- $\beta$ : the molecular Jekyll and Hyde of cancer. Nat Rev Cancer 2006; 6: 506-20.

19. Gilbert LA, Hemann MT. DNA damage-mediated induction of a chemoresistant niche. Cell 2010; 143: 355-66.

20. Gazdar AF. Activating and resistance mutations of EGFR in non-small-cell lung cancer: role in clinical response to EGFR tyrosine kinase inhibitors. Oncogene 2009; 28: S24-S31.

21. Katayama R, Khan TM, Benes C, et al. Therapeutic strategies to overcome crizotinib resistance in non-small cell lung cancers harboring the fusion oncogene EML4ALK. Proc Natl Acad Sci U S A 2011; 108: 7535-40. doi: $10.1073 /$ pnas.1019559108.

22. http://www.rosoncoweb.ru/news/oncology/2013/12/03/

23. Vtorushin SV, Khristenko KY, Zavyalova MV, et al. The phenomenon of multi-drug resistance in the treatment of malignant tumors. Exp Oncol 2014; 36: 144-56.

24. Wind NS, Holen I. Multidrug resistance in breast cancer: from in vitro models to clinical studies. Int $\mathrm{J}$ Breast Cancer 2011; 2011: 1-12.

25. Denisov EV, Gerashchenko TS, Zavyalova MV, et al. Invasive and drug resistant expression profile of different morphological structures of breast tumors. Neoplasma 2015; 62: 405-11.

26. Litviakov NV, Cherdyntseva NV, Tsyganov MM, et al. Deletions of multidrug resistance gene loci in breast cancer leads to the down-regulation of its expression and predict tumor response to neoadjuvant chemotherapy. Oncotarget 2016. doi: 10.18632/oncotarget.6953.

27. Litviakov NV, Cherdyntseva NV, Tsyganov MM, et al. Changing the expression vector of multidrug resistance genes is related to neoadjuvant chemotherapy response. Cancer Chemother Pharmacol 2013; 71: 153-63. doi: $10.1007 / \mathrm{s} 00280-012-1992-\mathrm{x}$.

28. Ilie M, Hofman V, Long E, et al. Current challenges for detection of circulating tumor cells and cell-free circulating nucleic acids, and their characterization in non-small cell lung carcinoma patients. What is the best blood substrate for personalized medicine? Ann Transl Med 2014; 2: 107. doi: 10.3978/j.issn.2305-5839.2014.08.11.

29. ESMO handbook of translational research. 2nd ed. G Tortora, C Sessa, A Scarpa, S Banerjee, eds. ESMO: Press, 2015. 133 p.

30. Cherdyntseva N, Litviakov N, Ivanova F, et al. The molecular aspects of personalized anticancer treatment. AIP Conf Proceed 2016; 1760: 020010. dx.doi.org/10.1063/1.4960229.

31. Gervas $P$, Ivanova $A$, Vasiliev $N$, et al. Frequency of EGFR mutations in non-small cell lung cancer patients: screening data from West Siberia. As Pac J Cancer Prevent 2015; 16: 689-92.

32. Taenzer A, Alix-Panabieres C, Wikman H, et al. Circulating tumor-derived biomarkers in lung cancer. $\mathrm{J}$ Thorac Dis 2012; 4: 448-9.
33. Meng S, Tripathy D, Shete S, et al. HER2 gene amplification can be acquired as breast cancer progresses. Proc Natl Acad Sci U S A 2004; 101: 9393-8.

34. Santamaria-Martínez A, Huelsken J. The niche under siege: novel targets for metastasis therapy. J Int Med 2013; 274: $127-36$.

35. Kraan J, Sleijfer S, Strijbos MH. External quality assurance of circulating tumor cell enumeration using the CellSearch ${ }^{\circledR}$ system: a feasibility study. Cytometry B Clin Cytom 2011; 80: 112-8.

36. Krishnamurthy S, Cristofanilli M, Singh B, et al. Detection of minimal residual disease in blood and bone marrow in early stage breast cancer. Cancer 2010; 116: 3330-7.

37. Muller V, Riethdorf S, Rack B, et al. Prognostic impact of circulating tumor cells assessed with the CellSearch System and AdnaTest Breast in metastatic breast cancer patients: the DETECT study. Breast Cancer Res 2012; 14: R118.

38. Pierga JY, Bonneton C, Vincent-Salomon A, et al. Clinical significance of immunocytochemical detection of tumor cells using digital microscopy inperipheral blood and bone marrow of breast cancer patients. Clin Cancer Res 2004; 10: $1392-400$.

39. Cheung KJ, Padmanaban V, Silvestri V, et al. Polyclonal breast cancer metastases arise from collective dissemination of keratin 14-expressing tumor cell clusters. Proc Natl Acad Sci U S A 2016; 113: E854-63.

40. Hou JM, Krebs MG, Lancashire L, et al. Clinical significance and molecular characteristics of circulating tumor cells and circulating tumor microemboli in patients with smallcell lung cancer. J Clin Oncol 2012; 30: 525-32.

41. Tsuji T, Ibaragi S, Shima K, et al. Epithelial-mesenchymal transition induced by growth suppressor p12CDK2AP1 promotes tumor cell local invasion but suppresses distant colony growth. Cancer Res 2008; 68: 10377-86.

42. Friedl P, Alexander S. Cancer invasion and the microenvironment: plasticity and reciprocity. Cell 2011; 147: 992-1009.

43. Kusters B, Kats G, Roodink I, et al. Micronodular transformation as a novel mechanism of VEGF-A-induced metastasis. Oncogene 2007; 26: 5808-15.

44. Aceto N, Bardia A, Miyamoto DT, et al. Circulating tumor cell clusters are oligoclonal precursors of breast cancer metastasis. Cell 2014; 158: 1110-22.

45. Al-Mehdi AB, Tozawa K, Fisher AB, et al. Intravascular origin of metastasis from the proliferation of endotheliumattached tumor cells: a new model for metastasis. Nat Med 2000; 6: 100-2.

46. Yu M, Bardia A, Wittner BS, et al. Circulating breast tumor cells exhibit dynamic changes in epithelial and mesenchymal composition. Science 2013; 339: 580-4.

47. Molnar B, Ladanyi A, Tanko L, et al. Circulating tumor cell clusters in the peripheral blood of colorectal cancer patients. Clin Cancer Res 2001; 7: 4080-5.

48. https://www.cellsearchctc.com/about-cellsearch/ how-cellsearch-ctc-test-works

49. Bednarz-Knoll N, Alix-Panabières C, Pantel K. Plasticity of disseminating cancer cells in patients with epithelial malignancies. Cancer Metastas Rev 2012; 31: 673-87.

50. Joosse SA, Pantel K. Biologic challenges in the detection of circulating tumor cells. Cancer Res 2013; 73: 8-11.

51. Balic M, Lin H, Williams A, et al. Progress in circulating tumor cell capture and analysis: implications for cancer management. Ezpert Rev Mol Diagnost 2012; 12: 303-12.

52. Howard H. Medical institute: genetic markers to guide targeted cancer therapies (http://www.hhmi.org/research/ genetic-markers-guide-targeted-cancer-therapies). 
53. Pukazhendhi G, Glück S. Circulating tumor cells in breast cancer. J Carcinogen 2014; 13: 8 .

54. Serrano MJ, Ortega FG, Alvarez-Cubero MJ, et al. EMT and EGFR in CTCs cytokeratin negative non-metastatic breast cancer. Oncotarget 2014; 5: 7486.

55. Kim MY, Oskarsson T, Acharyya S, et al. Tumor selfseeding by circulating cancer cells. Cell 2009; 139: 1315-26.

56. Schindlbeck C, Andergassen U, Hofmann $\mathrm{S}$, et al. Comparison of circulating tumor cells (CTC) in peripheral blood and disseminated tumor cells in the bone marrow (DTCBM) of breast cancer patients. J Cancer Res Clin Oncol 2013; 139: $1055-62$.

57. Barrière G, Riouallon A, Renaudie J, et al. Mesenchymal and stemness circulating tumor cells in early breast cancer diagnosis. BMC Cancer 2012; 12: 114.

58. Sarioglu AF, Aceto N, Kojic N, et al. A microfluidic device for label-free, physical capture of circulating tumor cell clusters. Nat Methods 2015; 12: 685-91.

59. Grosse-Wilde A, Fouquier d'Herouel A, McIntosh E, et al. Stemness of the hybrid epithelial/mesenchymal state in breast cancer and its association with poor survival. PLoS One 2015; 10: e0126522.

60. Krakhmal NV, Zavyalova MV, Denisov EV, et al. Cancer invasion: patterns and mechanisms. Acta Naturea 2015; 7: 17-28.

61. Lucci A, Hall CS, Lodhi AK, et al. Circulating tumour cells in non-metastatic breast cancer: a prospective study. Lancet Oncol 2012; 13: 688-95.

62. Van Dalum G, Van der Stam GJ, Tibbe AG, et al. Circulating tumor cells before and during follow-up after breast cancer surgery. Int J Oncol 2015; 46: 407-13.

63. Joyce JA, Pollard JW. Microenvironmental regulation of metastasis. Nat Rev Cancer 2009; 9: 239-52.

64. Cristofanilli M, Hayes DF, Budd GT, et al. Circulating tumor cells: a novel prognostic factor for newly diagnosed metastatic breast cancer. J Clin Oncol 2005; 23: 1420-30.

65. Papadaki MA, Kallergi G, Zafeiriou Z, et al. Co-expression of putative stemness and epithelial-to-mesenchymal transition markers on single circulating tumour cells from patients with early and metastatic breast cancer. BMC Cancer 2014; 14: 651.

66. Rack B, Schindlbeck C, Jückstock J, et al. Circulating tumor cells predict survival in early average-to-high risk breast cancer patients. J Natl Cancer Inst 2014; 106: dju066.

67. Lang JE, Mosalpuria $\mathrm{K}$, Cristofanilli $\mathrm{M}$, et al. HER2 status predicts the presence of circulating tumor cells in patients with operable breast cancer. Breast Cancer Res Treat 2009; 113: 501-7.

68. Ignatiadis $M$, Xenidis N, Perraki M, et al. Different prognostic value of cytokeratin-19 mRNA positive circulating tumor cells according to estrogen receptor and HER2 status in early-stage breast cancer. J Clin Oncol 2007; 25: 5194-202.

69. Agelaki S, Kalykakil A, Markomanolaki H, et al. Efficacy of lapatinib in therapy-resistant HER2-positive circulating tumor cells in metastatic breast cancer. PLoS One 2015; 10: $\mathrm{e} 0123683$.
70. Georgoulias V, Bozionelou V, Agelaki S, et al. Trastuzumab decreases the incidence of clinical relapses in patients with early breast cancer presenting chemotherapy-resistant CK-19mRNA-positive circulating tumor cells: results of a randomized phase II study. Ann Oncol 2012; 23: 1744-50.

71. Prat A, Baselga J. Dual human epidermal growth factor receptor 2 (HER2) blockade and hormonal therapy for the treatment of primary HER2-positive breast cancer: one more step toward chemotherapy-free therapy. J Clin Oncol 2013; 31: 1703-6.

72. Ignatiadis M, Pierga JY, Campion M, et al. CTCs and HER2-positive CTCs detection by CellSearch ${ }^{\circledR}$ in nonmetastatic breast cancer: an international ring study to assess inter-reader variability. Cancer Res 2011; 71: Abstr 4-07-14.

73. Pachmann K, Camara O, Kroll T, et al. Efficacy control of therapy using circulating epithelial tumor cells (CETC) as "liquid biopsy": Trastuzumab in HER2/neu-positive breast carcinoma. J Cancer Res Clin Oncol 2011; 137: 1317-27.

74. Liotta LA, Saidel MG, Kleinerman J. The significance of hematogenous tumor cell clumps in the metastatic process. Cancer Res 1976; 36: 889-94.

75. Mu Z, Wang C, Ye Z, et al. Prospective assessment of the prognostic value of circulating tumor cells and their clusters in patients with advanced-stage breast cancer. Breast Cancer Res Treat 2015; 154: 563-71.

76. Hou JM, Krebs MG, Lancashire L, et al. Clinical significance and molecular characteristics of circulating tumor cells and circulating tumor microemboli in patients with smallcell lung cancer. J Clin Oncol 2012; 30: 525-32.

77. Mallini P, Lennard T, Kirby J, Meeson A. Epithelialto-mesenchymal transition: what is the impact on breast cancer stem cells and drug resistance. Cancer Treat Rev 2014; 40: $341-8$

78. Denes V, Lakk M, Makarovskiy A, et al. Metastasis blood test by flow cytometry: in vivo cancer spheroids and the role of hypoxia. Int J Cancer 2015; 136: v1528-36.

79. Paget $\mathrm{S}$. The distribution of secondary growths in cancer of the breast. Cancer Metastasis Rev 1889; 8: 98-101.

80. Lyden D, Hattori K, Dias S, et al. Impaired recruitment of bone marrow derived endothelial and hematopoietic precursor cells blocks tumor angiogenesis and growth. Nat Med 2001; 7: 1194-201.

81. Sceneay J, Smyth MJ, Möller A. The pre-metastatic niche: finding common ground. Cancer Metastas Rev 2013; 32: $449-64$.

82. Perelmuter VM, Manskikh VN. Prenishe as the missing link of metastatic nishe concept explaining organ-preferential metastases formation and the type of metastatic disease. Biochemistry (Mosc) 2012; 77: 130-9.

83. Quail DF, Joyce JA. Microenvironmental regulation of tumor progression and metastasis. Nat Med 2013; 19: $1423-37$.

84. Wang X, Sun Q, Mao F, et al. CTCs hemodialysis: can it be a new therapy for breast cancer? Med Hypotheses 2013; 80: 99-101. 